\title{
Efeito do pré-condicionamento isquêmico remoto no consumo máximo de oxigênio e potência máxima em corredores e ciclistas: revisão sistemática e metanálise
}

\author{
Effect of remote ischemic pre-conditioning on maximum \\ oxygen consumption and maximum power in runners \\ and cyclists: systematic review and metanalysis
}

\author{
Francisco Tiago Oliveira de Oliveira' ${ }^{1}$ (I) \\ Marcos Eduardo Freitas ${ }^{2}$ (1) \\ Celso Nascimento Almeida ${ }^{3}$ (i)
}

\author{
Robson Santos Santana ${ }^{4}$ (1) \\ Ciro Oliveira Queiroz ${ }^{5}$ \\ Cristiane Maria Carvalho Costa Dias ${ }^{6}$ (1)
}

${ }^{1-5}$ Escola Bahiana de Medicina e Saúde Pública (Salvador). Bahia, Brasil. franciscooliveira@bahiana.edu.br, marcosfreitas.pos@bahiana.edu.br celsoalmeida15.2@bahiana.edu.br, robsonsantana.pos@bahiana.edu.br, ciroqueiroz@bahiana.edu.br

${ }^{6}$ Autora para correspondência. Escola Bahiana de Medicina e Saúde Pública (Salvador). Bahia, Brasil. cmccdias@bahiana.edu.br

\begin{abstract}
RESUMO | INTRODUÇão: O pré-condicionamento isquêmico remoto (PCIR) é uma intervenção cardioprotetora não invasiva que atenua a lesão celular sofrida por uma isquemia prolongada. Seus efeitos de proteção sobre o coração, quando aplicado ao esporte, pode melhorar o desempenho do exercício. OBJETIVO: Investigar o efeito do pré-condicionamento isquêmico remoto no consumo máximo de oxigênio (VO2máx) e potência máxima (Wmáx) em corredores e ciclistas. METODOLOGIA: Revisão sistemática e metanálise, com ensaios clínicos randomizados. Baseado no PRISMA e avaliado pelo repositório de projetos de revisões sistemática PROSPERO; entretanto, não obteve o registro por se tratar de um desfecho de performance esportiva. As buscas foram realizadas nas bases de dados Medline/PubMed, SciELO, Periódicos CAPES. A seleção dos estudos foi realizada em duas etapas: leitura do título e resumo, e leitura completa dos artigos. A extração dos dados foi realizada pela transcrição das informações. A qualidade metodológica foi avaliada pela escala risco de viés através da ferramenta Cochrane. Excluíram-se estudos que investigaram variáveis diferentes dos desfechos selecionados para esta revisão. RESULTADOS: Foram incluídos oito ensaios clínicos. Verificou-se que nos itens geração de sequência aleatória, ocultação de alocação e cegamento de avaliadores de desfecho em quase todos os estudos tiveram alto risco de viés. Os resultados da metanálise não mostraram diferenças significativas no VO2máx e Wmáx. CONCLUSÃO: O pré-condicionamento isquêmico remoto não se mostrou eficaz para aumentar o VO2máx e a Wmáx em corredores e ciclistas.
\end{abstract}

PALAVRAS-CHAVE: Aptidão Cardiorrespiratória. Exercício Físico. Pré-condicionamento Isquêmico.
ABSTRACT | INTRODUCTION: Remote ischemic preconditioning (PIRC) is a non-invasive cardioprotective intervention that attenuates cell damage suffered by prolonged ischemia. Its protective effects on the heart, when applied to sport, can improve exercise performance. OBJECTIVE: To investigate the effect of remote ischemic preconditioning on maximum oxygen consumption (VO2max) and maximum power (Wmax) in runners and cyclists. METHODOLOGY: Systematic review and metaanalysis, with randomized clinical trials. Based on PRISMA and evaluated by the PROSPERO systematic review project repository; however, it did not obtain registration because it is an outcome of sports performance. The searches were carried out in the Medline / PubMed, SciELO, Capes Periodicals databases. The selection of studies was carried out in two stages: reading the title and summary and reading the articles in full. Data extraction was performed by transcribing the information. Methodological quality was assessed by the risk of bias scale using the Cochrane tool. Studies that investigated variables other than the outcomes selected for this review were excluded. RESULTS: Eight clinical trials were included. In the generation of the item of random sequence, concealment of allocation and blinding of outcome evaluators in almost all studies had a high risk of bias. The analysis of the risk of bias was high risk. The results of the meta-analysis did not show significant differences in VO2max and Wmax. CONCLUSION: Remote ischemic preconditioning was not effective in increasing VO2max and Wmax in runners and cyclists.

KEYWORDS: Cardiorespiratory Fitness. Exercise. Ischemic Preconditioning.

Como citar este artigo: Oliveira FTO, Freitas ME, Almeida CN, Santana RS, Queiroz CO, Dias CMCC. Efeito do pré-condicionamento isquêmico remoto no consumo máximo de oxigênio e potência máxima em corredores e ciclistas: revisão sistemática e metanálise. Rev Pesqui Fisioter. 2021;11(2):435-444. Errata em: Rev Pesqui Fisioter. 2021;11(3):609-618. http://dx.doi.org/10.17267/2238-2704rpf. v11i3.3859 


\section{Introdução}

O pré-condicionamento isquêmico remoto (PCIR) é uma intervenção cardioprotetora não invasiva que envolve breves ciclos de isquemia, intercalados por reperfusão, que atenuam a lesão celular sofrida por uma isquemia prolongada do mesmo órgão. ${ }^{1}$ Foi descrito pela primeira vez em um estudo de Murry et al. em 1986, em que corações de cães foram pré-condicionados com quatro ciclos de oclusão e reperfusão. Verificou-se que a área de infarto do miocárdio nos corações pré-condicionados reduziu em $75 \%$ em comparação aos que não receberam o pré-condicionamento isquêmico. 2,3

O estímulo isquêmico proporcionado pelo PCIR leva à produção de substâncias que, entrando em circulação, atingem o miocárdio e outros tecidos, e consequentemente poderá exercer o efeito protetor. Essa proteção parece envolver a ativação da proteína quinase $C$ (PKC), através da cascata de sinalização de agonistas da proteína $\mathrm{G}$, com abertura subsequente de canais de potássio dependentes de ATP (KATP). ${ }^{4}$ Os efeitos de proteção do PCIR sobre o coração, além de ser benéfico no cenário clínico, pode também ser aplicado no esporte para melhorar o desempenho do exercício ${ }^{-5-8}$; esses efeitos foram analisados em atletas de endurance. Em teste de corrida de $5 \mathrm{~km}$, os indivíduos conseguiram completar a prova 34 segundos mais rápido e o aumento do lactato foi atenuado em 1,07 $\pm 0,11$ mmol durante um teste de corrida incremental, após o PCIR. ${ }^{9}$ Em nadadores treinados, o PCIR foi associado ao ganho do desempenho máximo, com uma redução no tempo de natação de $100 \mathrm{~m}$ e um melhor tempo de natação em relação ao tempo pessoal. 10

O desempenho esportivo é objeto de estudo das mais diversas áreas do conhecimento e almejado por atletas, treinadores, fisiologistas, entre outros. Nos últimos anos, muitas técnicas têm sido estudadas para otimizar a performance e resultados de atletas de todas as modalidades. Dentre essas técnicas, destacase o PCIR, que favorece o processo de angiogênese e maior resistência a isquemia tissular. 11 Modalidades esportivas como a corrida e o ciclismo que têm predominância aeróbica promovem adaptações capazes de gerar aumentos do VO2máx, e os ajustes fisiológicos que promovem modificações funcionais em resposta ao treinamento de endurance só são possíveis através da manipulação das variáveis da capacidade aeróbica, independente da modalidade praticada. ${ }^{12}$
O PCIR é uma estratégia não invasiva e de fácil aplicação que pode promover a melhora do desempenho do exercício. ${ }^{13,14}$ Dessa forma, o objetivo deste estudo é investigar o efeito do pré-condicionamento isquêmico remoto no consumo máximo de oxigênio (VO2máx) e potência máxima (Wmáx) em corredores e ciclistas.

\section{Metodologia}

Trata-se de uma revisão sistemática e metanálise. Foi baseada pelo "The PRISMA 2020 statement: An updated guideline for reporting systematic reviews" 15 , e avaliada pelo repositório de projetos de revisões sistemática PROSPERO, entretanto, não obteve o registro por se tratar de um desfecho de performance esportiva. Incluíram-se ensaios clínicos randomizados que investigaram o efeito do pré-condicionamento isquêmico remoto no VO2máx e Wmáx de corredores e ciclistas. Foram excluídos os estudos que não avaliaram desfechos de interesse desta revisão.

A seleção dos estudos foi realizada por dois pesquisadores independentes com a mesma estratégia de busca, em caso de discordância, um terceiro pesquisador foi acionado. Não foi utilizado software para auxiliar no processo de seleção. Posteriormente, os artigos selecionados foram avaliados o risco de viés através da ferramenta Cochrane. ${ }^{16} \mathrm{~A}$ busca foi realizada através da estratégia PICOS nas bases de dados Medline/PubMed, SciELO, Periódicos Capes e busca manual, sem restrição do ano de publicação, disponíveis em português, inglês e espanhol. Não foram utilizados filtros nas bases de dados. A última busca foi realizada em uma única data 18 de novembro de 2020, em todas as bases de dados. Utilizou-se MeSH e o DeCS para encontrar os descritores e seus sinônimos. Os descritores incluídos na estratégia de busca para base de dados Medline/ PubMed foram: ( ( ((Ischemic Preconditioning) OR (remote ischemic preconditioning)) AND (exercise)) OR (Cardiorespiratory Fitness)) OR (exercise trainning)) AND (Clinical Trial). Para as bases de dados SciELO e Periódicos Capes foi utilizado: Ischemic preconditioning and exercise. Essa estratégia foi usada com o objetivo de realizar uma busca mais sensível do que especifica, considerando que essas bases de dados ainda têm uma produção científica relativamente baixa sobre a temática estudada. 
A seleção dos estudos foi realizada em duas etapas. A primeira etapa foi baseada em títulos e resumos; segunda etapa foi a análise do texto completo. Realizou-se a extração dos dados através da transcrição das informações e construiu-se um fichamento, contendo: autores e ano de publicação, participantes, intervenção, desfechos e desenho do estudo. Para apresentação do fluxograma foi seguido as orientações atualizadas do PRISMA. $\frac{15}{15}$ A qualidade dos estudos foi avaliada através da escala de risco de viés pela ferramenta da colaboração Cochrane. ${ }^{16}$

O software Revman 5.3 foi utilizado para elaboração, análise de dados e construção do gráfico Forest Plot. Avaliou-se a heterogeneidade estatística pela inspeção visual do intervalo de confiança, pelo teste Q-Cochran e teste qui-quadrado (X2). Após esta avaliação, os dados foram analisados através do modelo de efeito randômico, na expectativa de heterogeneidade clínica e metodológica entre os estudos. Todos os dados foram analisados com a mesma medida, mililitros por minutos, os estudos que apresentaram o VO2máx em litros por minutos; foi realizada a transformação para mililitros por minutos.

\section{Resultados}

\section{Seleção dos estudos}

O processo de busca e identificação dos estudos inseridos na revisão estão resumidos na Figura 1. Foi identificado um total de 1383 estudos. Após leitura de título e resumo, 1356 foram excluídos e outros 27 foram selecionados para leitura de texto completo. Após análise, 8 estudos preencheram os critérios de elegibilidade, permanecendo na revisão sistemática para análise.

Figura 1. Fluxograma do processo de busca e identificação dos estudos

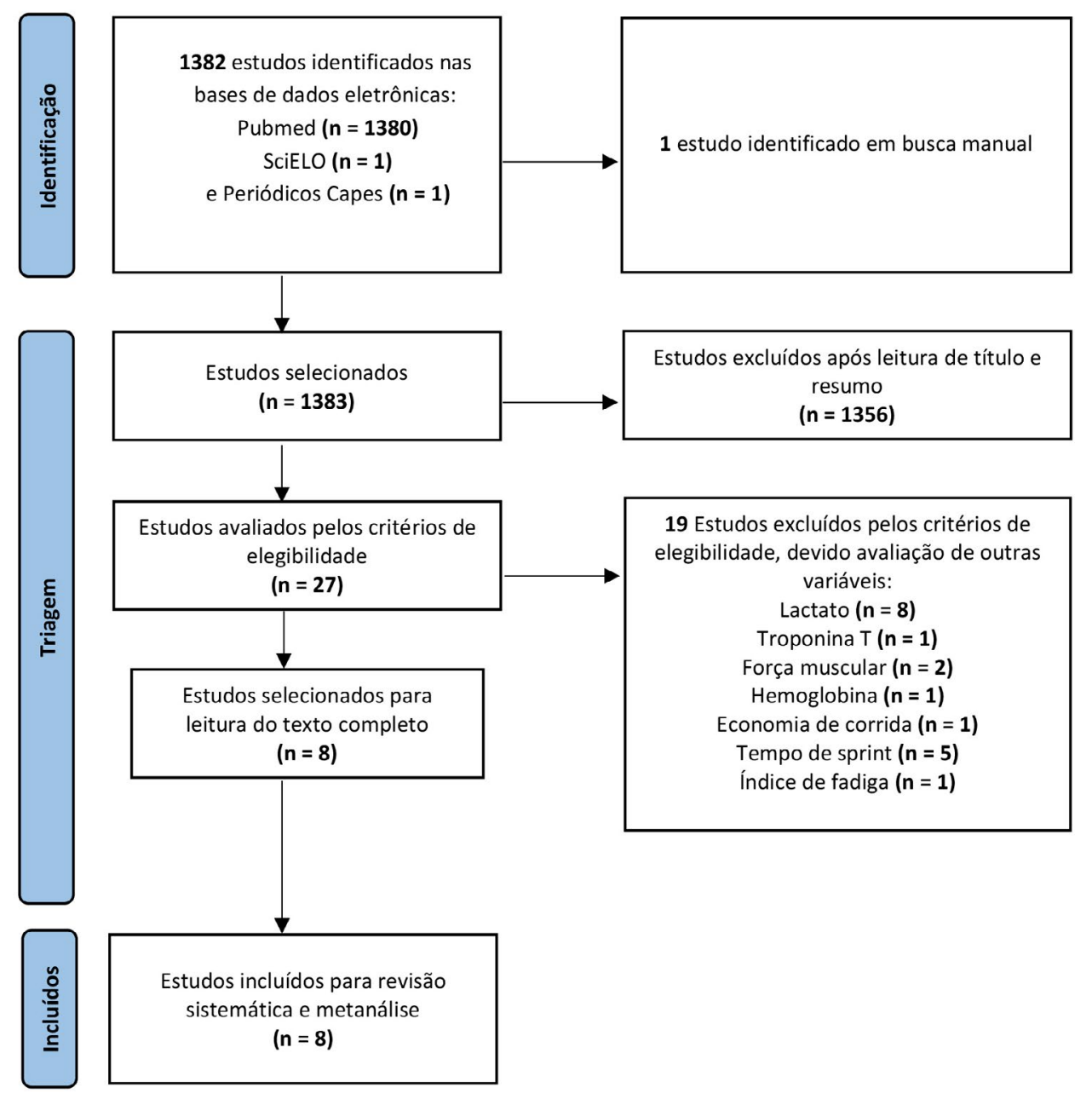




\section{Características dos estudos}

A Tabela 1 resume as características descritivas dos ensaios clínicos incluídos na revisão sistemática, compreendendo um total de 107 indivíduos do sexo masculino e

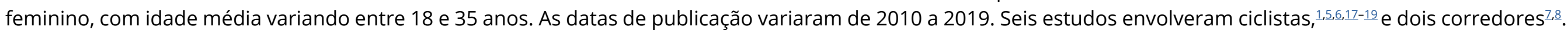
Cada estudo utilizou diferentes tipos de protocolos na avaliação do VO2máx e da Wmáx. O protocolo do PCIR foi semelhante entre os estudos, entretanto houve variação do número de ciclos de isquemia-reperfusão.

Tabela 1. Características dos ensaios clínicos incluídos na revisão sistemática. Efeito do pré-condicionamento isquêmico remoto no VO2máx e Wmáx de corredores e ciclistas, 2019/2020. N=8 (continua)

\begin{tabular}{|c|c|c|c|c|c|}
\hline Estudo & Participantes & Intervenção & Comparação & Desfechos & Desenho de Estudo \\
\hline $\begin{array}{l}\text { Autores e } \\
\text { ano de } \\
\text { publicação }\end{array}$ & $\begin{array}{l}\text { Número no Grupo Controle } \\
\text { (nGC); Número no Grupo Pré- } \\
\text { condicionamento isquêmico } \\
\text { (nPCI) }\end{array}$ & $\begin{array}{l}\text { Modalidade esportiva (Esporte); } \\
\text { Tipo exercício (Exercício) }\end{array}$ & $\begin{array}{l}\text { Protocolo grupo Controle (CT); } \\
\text { Protocolo grupo Pré- } \\
\text { condicionamento isquêmico } \\
\text { (PCI) }\end{array}$ & $\begin{array}{l}\text { Consumo máximo de oxigênio } \\
\text { (VO2máx) e Potência máxima em } \\
\text { watts (Wmáx) }\end{array}$ & Tipo ECR \\
\hline $\begin{array}{l}\text { Patterson } \\
\text { et al., } 2015^{6}\end{array}$ & $\begin{array}{l}\text { nGC: } 14 ; \text { nPCI: } 14 ; \mathbf{I}: 22,9 \pm 3,7 \\
\text { H: } 14\end{array}$ & $\begin{array}{l}\text { Esporte: Ciclismo } \\
\text { Exercício: } 12 \text { sprints de } 6 " \text { com } \\
\text { descanso de } 30 " \text { entre cada sprint. }\end{array}$ & $\begin{array}{l}\text { PCI: } 4 \times 5^{\prime} \text { oclusão / 5' } \\
\text { reperfusão - } 220 \text { mmHg. } \\
\text { CT: } 4 \times 5^{\prime} \text { oclusão / 5' reperfusão } \\
\text { - 20mmHg. }\end{array}$ & $\begin{array}{l}\text { VO2máx PCl: 2,7 } \pm 0,4 \text { L.min } \\
\text { VO2máx CT: } 2,6 \pm 0,3 \text { L.min }\end{array}$ & $\begin{array}{l}\text { Randomizado, } \\
\text { duplo-cego, cruzado }\end{array}$ \\
\hline $\begin{array}{l}\text { Crisafulli et } \\
\text { al., 2011' }\end{array}$ & $\begin{array}{l}\text { nGC: } 17 ; \text { nPCI: } 17 ; \mathbf{I}: 35,2 \pm 9,1 \\
\text { H: } 17\end{array}$ & $\begin{array}{l}\text { Esporte: Ciclismo } \\
\text { Exercício: Teste } \\
\text { incremental em ciclo ergômetro } \\
\text { com aumento linear de carga de } \\
\text { trabalho de } 25 \mathrm{~W} / \text { min. }\end{array}$ & $\begin{array}{l}\text { CT: Sem intervenção } \\
\text { PCl: } 3 \times \text { 5' oclusão / 5' reperfusão } \\
-220 \text { mmHg. }\end{array}$ & $\begin{array}{l}\text { VO2máx CT: } 2963,8 \pm 256,6(\mathrm{ml} / \mathrm{min}) \\
\text { VO2máx PCl: } 3068,3 \pm 396,1 \\
\text { (ml/min) } \\
\text { Wmáx CT: } 277,9 \pm 44 \mathrm{~W} \\
\text { Wmáx PCl: } 288,2 \pm 47,6 \mathrm{~W}\end{array}$ & $\begin{array}{l}\text { Randomizado, } \\
\text { cruzado }\end{array}$ \\
\hline
\end{tabular}


Tabela 1. Características dos ensaios clínicos incluídos na revisão sistemática. Efeito do pré-condicionamento isquêmico remoto no VO2máx e Wmáx de corredores e ciclistas, 2019/2020. N=8 (continuação)

\begin{tabular}{|c|c|c|c|c|c|}
\hline Estudo & Participantes & Intervenção & Comparação & Desfechos & Desenho de Estudo \\
\hline $\begin{array}{l}\text { Autores e } \\
\text { ano de } \\
\text { publicação }\end{array}$ & $\begin{array}{l}\text { Número no Grupo Controle } \\
\text { (nGC); Número no Grupo Pré- } \\
\text { condicionamento isquêmico } \\
\text { (nPCI) }\end{array}$ & $\begin{array}{l}\text { Modalidade esportiva (Esporte); } \\
\text { Tipo exercício (Exercício) }\end{array}$ & $\begin{array}{l}\text { Protocolo grupo Controle (CT); } \\
\text { Protocolo grupo Pré- } \\
\text { condicionamento isquêmico } \\
\text { (PCI) }\end{array}$ & $\begin{array}{l}\text { Consumo máximo de oxigênio } \\
\text { (Vo2máx) e Potência máxima em } \\
\text { watts (Wmáx) }\end{array}$ & Tipo ECR \\
\hline $\begin{array}{l}\text { de Groot et } \\
\text { al., 201017 }\end{array}$ & $\begin{array}{l}\text { nGC: } 15 \\
\text { nPCl: } 15 \\
\text { I: } 27,2 \pm 5,6 \\
\text { H: } 12 \\
\text { M: } 3\end{array}$ & $\begin{array}{l}\text { Esporte: Ciclismo } \\
\text { Exercício: Teste } \\
\text { incremental máximo em ciclo } \\
\text { ergômetro com aumento da } \\
\text { carga até exaustão. }\end{array}$ & $\begin{array}{l}\text { CT: Sem intervenção } \\
\text { PCl: } 3 \times 5 \text { ' oclusão / 5' reperfusão } \\
-220 \text { mmHg. }\end{array}$ & $\begin{array}{l}\text { VO2máx CT: } 56,8 \pm 6,8(\mathrm{ml} / \mathrm{min} / \mathrm{kg}) \\
\text { VO2máx PCl: } 58,4 \pm 6,2(\mathrm{ml} / \mathrm{min} / \mathrm{kg}) \\
\text { Wmáx CT: } 366 \pm 62 \mathrm{~W} \\
\text { Wmáx PCI: } 372 \pm 59 \mathrm{~W}\end{array}$ & $\begin{array}{l}\text { Randomizado, } \\
\text { cruzado }\end{array}$ \\
\hline $\begin{array}{l}\text { Paradis- } \\
\text { Deschênes } \\
\text { et al., 2017'18 }\end{array}$ & $\begin{array}{l}\text { nGC: } 13 \\
\text { nPCl: } 13 \\
\text { I: } 27,5 \pm 1,6 \\
\text { H: } 13\end{array}$ & $\begin{array}{l}\text { Esporte: Ciclismo } \\
\text { Exercício: Testes de tempo de } \\
\text { ciclismo de } 5 \mathrm{~km} \text { com simulação } \\
\text { de altitude moderada. }\end{array}$ & $\begin{array}{l}\text { CT: Manguito inflado a } 20 \\
\text { mmHg. } \\
\text { PCl: } 3 \times 5 \text { ' oclusão / 5' reperfusão } \\
\text { - } 220 \text { mmHg. }\end{array}$ & $\begin{array}{l}\text { Wmáx CT: } 284,6 \pm 12,7 \mathrm{~W} \\
\text { Wmáx PCI: } 291,3 \pm 9,8 \mathrm{~W}\end{array}$ & $\begin{array}{l}\text { Randomizado, } \\
\text { cruzado }\end{array}$ \\
\hline $\begin{array}{l}\text { Slysz et al., } \\
2019^{8}\end{array}$ & $\begin{array}{l}\text { nGC: } 7 ; \text { nPCI: } 7 \\
\text { I GC: } 19 \pm 2 ; \text { I PCI: } 18 \pm 1 \\
\text { H: } 11 ; \text { M:3 }\end{array}$ & $\begin{array}{l}\text { Esporte: Corrida } \\
\text { Exercício: Teste incremental } \\
\text { contínuo e teste de tempo de } 1 \\
\mathrm{~km} \\
\end{array}$ & $\begin{array}{l}\text { CT: Sem PCIR; PCI: } 3 \times 5 \text { ' oclusão } \\
\text { / 5' reperfusão - acima da } \\
\text { pressão arterial sistólica até } \\
\text { completa oclusão arterial. }\end{array}$ & $\begin{array}{l}\text { VO2máx CT: } 54,2 \pm 4(\mathrm{ml} / \mathrm{min} / \mathrm{kg}) \\
\text { VO2máx PCI: } 55,5 \pm 2(\mathrm{ml} / \mathrm{min} / \mathrm{kg})\end{array}$ & Randomizado \\
\hline $\begin{array}{l}\text { Kilding et } \\
\text { al., 2018 }\end{array}$ & $\begin{array}{l}\text { nGC: } 8 \\
\text { nPCl: } 8 \\
\text { I: } 27,0 \pm 7,0 \\
\text { H: } 8\end{array}$ & $\begin{array}{l}\text { Esporte: Ciclismo } \\
\text { Exercício: Teste de tempo } \\
\text { individual de } 4 \mathrm{~km} \text { e teste de } \\
\text { esforço em rampa. }\end{array}$ & $\begin{array}{l}\text { CT: } 4 \text { × 5' oclusão / 5' reperfusão } \\
-50 \text { mmHg. } \\
\text { PCl: } 4 \text { × 5' oclusão / 5' reperfusão } \\
\text { - } 200 \text { mmHg. }\end{array}$ & $\begin{array}{l}\text { VO2máx CT: 4,4 } \pm 0,6 \text { (L. min) } \\
\text { vo2máx PCl: 4,4 } \pm 0,5 \text { (L. min) } \\
\text { Wmáx CT: } 383 \pm 46 \mathrm{~W} \\
\text { Wmáx PCl: } 385 \pm 42 \mathrm{~W}\end{array}$ & $\begin{array}{l}\text { Randomizado, } \\
\text { cruzado }\end{array}$ \\
\hline $\begin{array}{l}\text { Sabino- } \\
\text { Carvalho et } \\
\text { al, 2017 }\end{array}$ & $\begin{array}{l}\text { nGC: } 16 ; \text { nPCI: } 16 \\
\text { I: } 22,3 \pm 0,9 ; \\
\text { GT: } 16\end{array}$ & $\begin{array}{l}\text { Esporte: Corrida } \\
\text { Exercício: Teste incremental em } \\
\text { esteira com aumento da carga a } \\
\text { cada estágio de 3' até exaustão. }\end{array}$ & $\begin{array}{l}\text { CT: Simulação de Ultra } \\
\text { sonografia } \\
\text { PCl: } 4 \times 5 \text { ' oclusão / 5' reperfusão } \\
\text { - } 220 \mathrm{mmHg} \text {. }\end{array}$ & $\begin{array}{l}\text { VO2máx CT: } 64,6 \pm 7,2(\mathrm{ml} / \mathrm{min} / \mathrm{kg}) \\
\text { VO2máx PCI: } 64,8 \pm 6,4(\mathrm{ml} / \mathrm{min} / \mathrm{kg})\end{array}$ & $\begin{array}{l}\text { Randomizado, } \\
\text { cruzado }\end{array}$ \\
\hline $\begin{array}{l}\text { Jeffries } \\
\text { et al., } 2019^{5}\end{array}$ & $\begin{array}{l}\text { nGC: } 10 ; \text { nPCI: } 10 \\
\text { I GC: } 21 \pm 2 ; \text { I PCI: } 22 \pm 3 \\
\text { H: } 20\end{array}$ & $\begin{array}{l}\text { Esporte: Ciclismo } \\
\text { Exercício: Teste incremental } \\
\text { com incremento de } 30 \mathrm{~W} \text { min até } \\
\text { exaustão. }\end{array}$ & $\begin{array}{l}\text { CT: } 4 \text { × 5' oclusão / 5' reperfusão } \\
-20 \mathrm{mmHg} \text {. } \\
\text { PCI: } 4 \times \text { × oclusão / 5' reperfusão } \\
-220 \mathrm{mmHg} \text {. }\end{array}$ & $\begin{array}{l}\text { VO2máx CT: 40,6 } 68,1(\mathrm{ml} / \mathrm{min} / \mathrm{kg}) \\
\text { VO2máx PCl: } 45 \pm 5,6(\mathrm{ml} / \mathrm{min} / \mathrm{kg}) \\
\text { Wmáx CT: } 292 \pm 35 \mathrm{~W} \\
\text { Wmáx PCI: } 323 \pm 51 \mathrm{~W}\end{array}$ & $\begin{array}{l}\text { Randomizado, } \\
\text { simples cego }\end{array}$ \\
\hline
\end{tabular}

: Idade média e desvio padrão; IGC: idade média grupo controle e desvio padrão; I PCl: idade média grupo pré-condicionamento isquêmico e desvio padrão; H: participantes sexo masculino;

M: participantes sexo feminino

Km: quilômetros; W: watts; W.min: watts por minuto; $\mathbf{m m H g}$ : milímetros de mercúrio; CT: grupo controle; GT: Grupo Total; PCI: grupo pré-condicionamento isquêmico; ml/min /kg: mililitros por minuto por quilo; L.min: litros por minuto; ml.min: mililitros por minuto; W: watts; ECR: Ensaio clínico randomizado. 


\section{Análise do Risco de viés}

Verificou-se que nos itens geração de sequência aleatória, ocultação de alocação e cegamento de avaliadores de desfecho em quase todos os estudos tiveram alto risco de viés, isso aconteceu pois os autores não apresentaram ou realizaram essas etapas nos estudos. Para os itens cegamento de participantes e profissionais, desfechos incompletos e outras fontes de viés cerca de metade dos estudos tiveram alto risco ou risco incerto. Somente o item relato de desfecho seletivo foi o que teve baixo risco de viés, pois, os protocolos dos estudos e seus desfechos primários foram reportados em detalhes, Figura 2.

Figura 2. Avaliação do risco de viés dos 8 estudos incluídos na revisão, através da escala risco de viés pela ferramenta da colaboração Cochrane. N=8

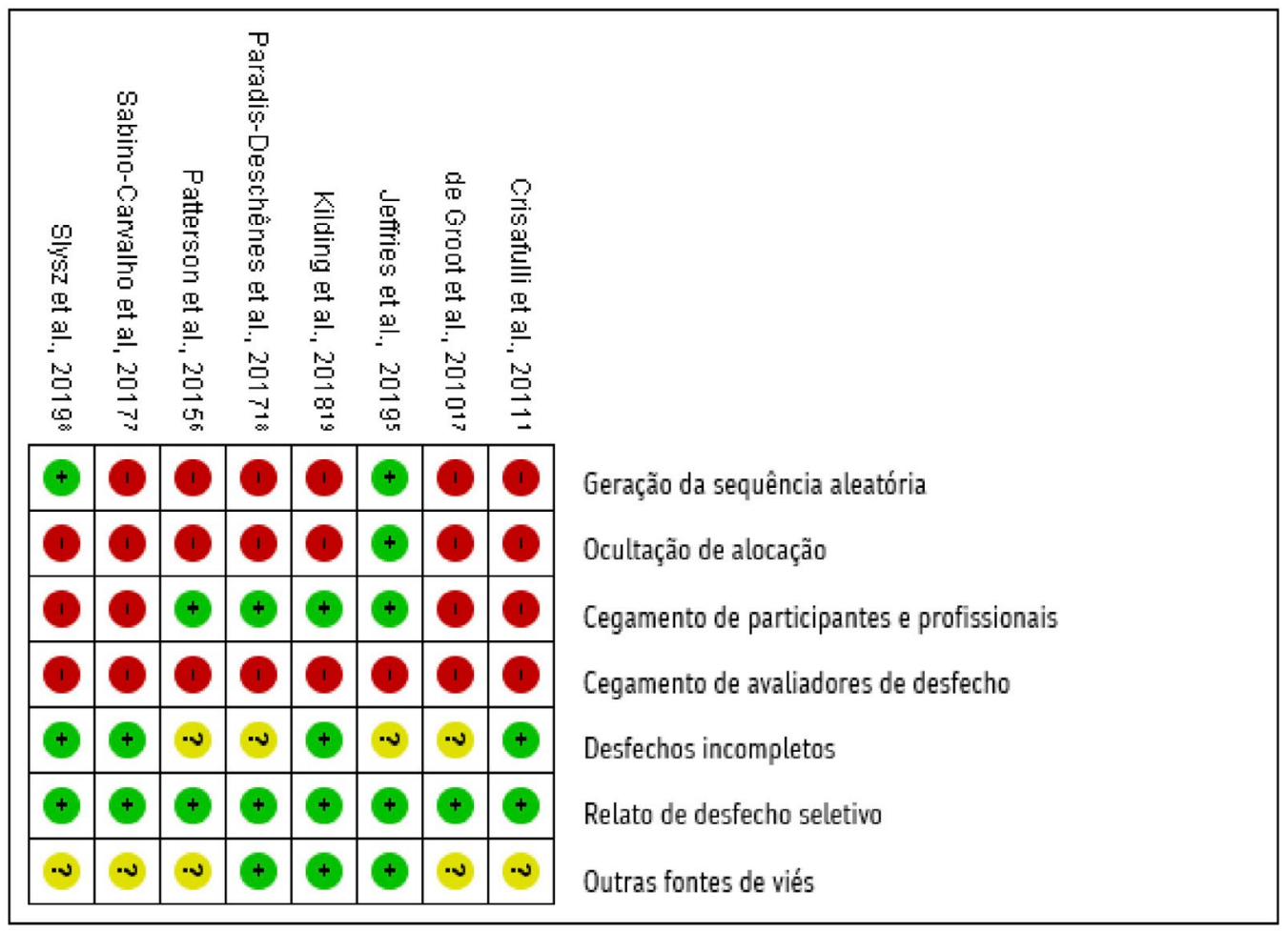

Baixo risco de viés

Risco de viés incerto

Alto risco de viés

\section{Resultados dos desfechos}

\section{Consumo máximo de oxigênio (VO2máx)}

Os estudos que avaliaram o efeito do pré-condicionamento isquêmico remoto no VO2máx, 1,5-8,17,19 continham um total de 87 participantes. Não foi observado um incremento significativo no VO2máx para os grupos que utilizaram protocolo de PCIR em comparação com os grupos controle, indicado na Figura 3. 
Figura 3. Forest Plot da comparação do VO2máx entre grupo controle e PCIR

\begin{tabular}{|c|c|c|c|c|c|c|c|c|c|}
\hline \multirow[b]{2}{*}{ Study or Subgroup } & \multicolumn{3}{|c|}{ PCIR } & \multicolumn{3}{|c|}{ Controle } & \multirow[b]{2}{*}{ Weight } & \multirow{2}{*}{$\begin{array}{l}\text { Mean Difference } \\
\text { IV, Random, } 95 \% \mathrm{Cl}\end{array}$} & \multirow{2}{*}{$\begin{array}{l}\text { Mean Difference } \\
\text { IV, Random, } 95 \% \mathrm{Cl}\end{array}$} \\
\hline & Mean & SD & Total & Mean & SD & Total & & & \\
\hline Crisafulli et al., $2011^{1}$ & 41.75 & 5.39 & 17 & 40.32 & 3.49 & 17 & $25.3 \%$ & $1.43[-1.62,4.48]$ & \\
\hline de Groot et al., 201017 & 58.4 & 6.2 & 15 & 56.8 & 6.8 & 15 & $10.9 \%$ & $1.60[-3.06,6.26]$ & \\
\hline Jeffries et al., $2019^{5}$ & 45 & 5.6 & 10 & 40.6 & 8.1 & 10 & $6.3 \%$ & $4.40[-1.70,10.50]$ & \\
\hline Kilding et al., 201819 & 57.44 & 6.53 & 8 & 57.44 & 7.83 & 8 & $4.7 \%$ & $0.00[-7.07,7.07]$ & \\
\hline Patterson et al., $2015^{6}$ & 34.93 & 5.17 & 14 & 33.64 & 3.88 & 14 & $20.6 \%$ & $1.29[-2.10,4.68]$ & \\
\hline Sabino-Cawalho et al, $2017^{7}$ & 64.8 & 6.4 & 16 & 64.6 & 7.2 & 16 & $10.6 \%$ & $0.20[-4.52,4.92]$ & \\
\hline Slysz et al., $2019^{\circ}$ & 55.5 & 2 & 7 & 54.2 & 4 & 7 & $21.5 \%$ & $1.30[-2.01,4.61]$ & \\
\hline Total $(95 \% \mathrm{Cl})$ & & & 87 & & & 87 & $100.0 \%$ & $1.38[-0.15,2.92]$ & \\
\hline \multicolumn{9}{|c|}{$\begin{array}{l}\text { Heterogeneity: } \operatorname{Tau}^{2}=0.00 ; \mathrm{Chi}^{2}=1.34, \mathrm{df}=6(\mathrm{P}=0.97) ; \mathrm{I}^{2}=0 \% \\
\text { Test for overall effect: } Z=1.76(\mathrm{P}=0.08)\end{array}$} & $\begin{array}{ccccc}-20 & -10 & 1 & 1 & 1 \\
\text { Favor [CONTROLE] } & \text { Favor [PCIR] }\end{array}$ \\
\hline
\end{tabular}

\section{Potência máxima (Wmáx)}

Cinco estudos $1,5,17-19$, com um total de 63 participantes, avaliaram o efeito do pré-condicionamento isquêmico remoto na Wmáx. Não foi observado uma diferença significativa quando comparado o grupo controle com o PCIR: 7.79 (IC95\% -0.12; 15.71), indicado na Figura 4.

Figura 4. Forest Plot da comparação da Wmáx entre grupo controle e PCIR

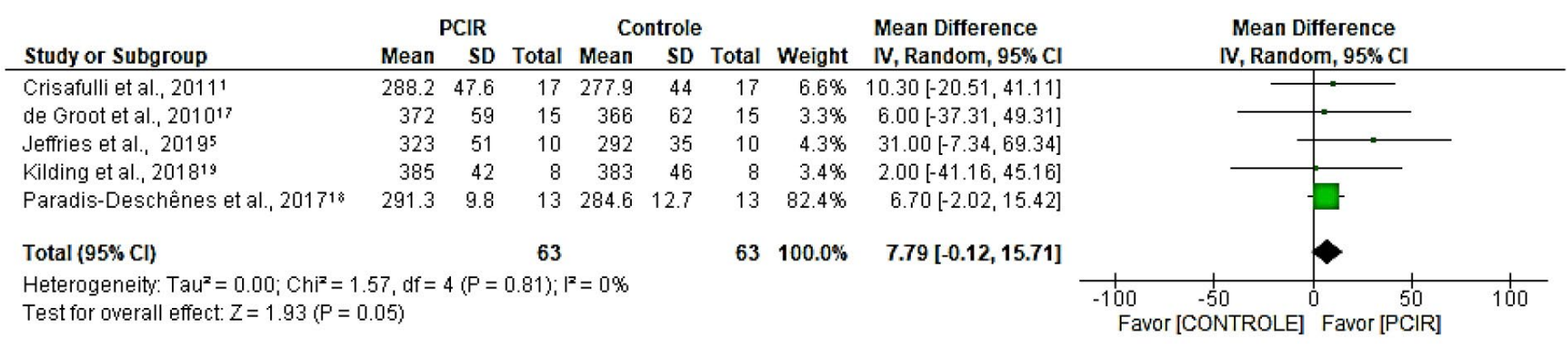

\section{Discussão}

Essa metanálise é original em testar os efeitos do pré-condicionamento isquêmico remoto nos indicadores de desempenho em corredores e ciclistas. Os resultados indicam que uma sessão de PCIR não foi capaz de induzir incremento agudo no VO2máx e na Wmáx.

O aumento do VO2máx é almejado por atletas, um preditor de performance em provas de contra relógio, além de ser o melhor índice para avaliação da aptidão cardiorrespiratória. 20,21 Os achados desta metanálise não corroboram com os resultados relatados por outros pesquisadores que observaram incremento do VO2máx quando utilizaram o PCIR antes do teste de desempenho máximo em ciclistas e corredores.

Vale ressaltar que o protocolo do PCIR utilizado por todos os pesquisadores foram semelhantes, variando pouco o número de ciclos de isquemia-reperfusão. . $15-8,17-19 \mathrm{~A}$ manobra consistia em períodos de isquemia de um membro, através da insuflação de um manguito até $220 \mathrm{mmHg}$ por 5 minutos, seguidos por 5 minutos de reperfusão com a deflação do manguito. Entretanto, os métodos de intervenção foram heterogêneos quanto as variáveis de treinamento, essas diferenças nos protocolos são essenciais na aplicabilidade prática, já que a manipulação de variáveis pode resultar na melhora do desempenho em atletas de endurance. 
Nesta revisão, foram incluídas duas modalidades esportivas, corredores e ciclistas, baseadas na comprovação que atletas de endurance, em particular, apresentam VO2máx similares. É geralmente descrito que o exercício aeróbico com a mesma intensidade induz uma maior absorção de oxigênio e, provavelmente, um gasto de energia semelhantes entre essas duas modalidades; outra justificativa é o padrão do recrutamento de unidades motoras relacionadas às tarefas executadas, com impacto no VO2máx. ${ }^{23}$

É essencial apontar que os estudos $1,5-8,17,19$ que avaliaram o VO2máx foram compostos por pequenas amostras de atletas com alta capacidade funcional, podendo enviesar, desta forma, os resultados encontrados. Com isso, as análises dos resultados podem apresentar uma baixa confiança, sendo suscetíveis a uma baixa validade externa.

O pré-condicionamento isquêmico remoto provoca a liberação de várias substâncias pelo tecido isquêmico, embora não esteja bem definido quais as substâncias. ${ }^{4}$ Estas podem influenciar na abertura de canais de potássio $(\mathrm{K}+)$ dependentes de ATP e liberação local de adenosina, além da regulação da via do óxido nítrico, sendo estes potentes vasodilatadores. ${ }^{24,25}$ Especula-se que a ação vasodilatadora desses mecanismos, participem nos efeitos do PCIR, e consequentemente no aumento do desempenho físico de atletas. ${ }^{17,25}$

Os estudos que avaliaram a Wmáx fizeram num teste incremental máximo em cicloergômetro. Nos resultados da metanálise, o PCIR não exerceu um efeito significativo no incremento da Wmáx. Os achados desta pesquisa coadunam com outros autores que avaliaram os efeitos do PCIR sobre a Wmáx em atletas. ${ }^{18,19}$ Estes resultados podem ser explicados pela baixa variabilidade da Wmáx em atletas de alto rendimento, sendo esta, uma variável mecânica com baixa sensibilidade para detectar alterações fisiológicas agudas, potencializado pelo intervalo de confiança amplo em todas as análises.

Como perspectiva, seria relevante estudos que avaliem o efeito de um período de treinamento com précondicionamento isquêmico remoto para avaliação de efeitos crônicos desta intervenção em esportes de endurance. As limitações dessa revisão sistemática se referem a diversidade dos protocolos de intervenção aplicada nos estudos, provavelmente essa característica pode ter influenciado nos achados.

\section{Conclusão}

O pré-condicionamento isquêmico remoto não influenciou no aumento do VO2máx e da Wmáx em praticantes de ciclismo e corrida.

\section{Agradecimentos}

Escola Bahiana de Medicina e Saúde Pública, Programa de PósGraduação de Tecnologias em Saúde e Grupo de Pesquisa de Fisioterapia Cardiovascular e Respiratória (GEPFIR).

Iza Cristina Salles de Castro (ORCID: 0000-0001-8514-7631) e Centro Internacional de Pesquisa e Medicina Clínica do Sono da BAHIANA - Escola de Medicina e Saúde Pública.

\section{Contribuições dos autores}

Oliveira FTO participou da concepção, desenho da obra e aquisição, análise ou interpretação dos dados da obra, e revisão crítica. Dias CMCC contribuiu no desenho da obra e aquisição, e revisão crítica. Queiroz C participou da revisão da análise/ interpretação dos dados da obra, revisão crítica. Freitas ME participou da busca dos dados e redação do manuscrito. Santana RS e Nascimento C participaram da coleta dos dados. Salles C participou da análise crítica do artigo.

\section{Conflitos de interesses}

Nenhum conflito financeiro, legal ou político envolvendo terceiros (governo, empresas e fundações privadas, etc.) foi declarado para nenhum aspecto do trabalho submetido (incluindo, mas não se limitando a subvenções e financiamentos, participação em conselho consultivo, desenho de estudo, preparação de manuscrito, análise estatística, etc.).

\section{Referências}

1. Crisafulli A, Tangianu F, Tocco F, Concu A, Mameli O, Mulliri G, et al. Ischemic preconditioning of the muscle improves maximal exercise performance but not maximal oxygen uptake in humans. J Appl Physiol. 2011;111(2):530-6. https://doi.org/10.1152/ japplphysiol.00266.2011

2. Sharma V, Marsh R, Cunniffe B, Cardinale M, Yellon DM, Davidson SM. From Protecting the Heart to Improving Athletic Performance - the Benefits of Local and Remote Ischaemic Preconditioning. Cardiovasc Drugs Ther. 2015;29(6):573-88. https://dx.doi.org/10.1007\%2Fs10557-015-6621-6 
3. Murry CE, Jennings RB, Reimer KA. Preconditioning with ischemia: A delay of lethal cell injury in ischemic myocardium. Circulation. 1986;74(5):1124-36. https://doi.org/10.1161/01. cir.74.5.1124

4. Costa JF, Fontes-carvalho R, Leite-moreira AF. Précondicionamento isquémico remoto do miocárdio: dos mecanismos fisiopatológicos à aplicacão na prática clínica. Rev Port Cardiol. 2013;32(11):893-904. https://doi.org/10.1016/j. repc.2013.02.012

5. Jeffries O, Evans DT, Waldron M, Coussens A, Patterson SD. Seven-day ischaemic preconditioning improves muscle efficiency during cycling. J Sports Sci. 2019;37(24):2798-805. https://doi.org/ 10.1080/02640414.2019.1664537

6. Patterson SD, Bezodis NE, Glaister M, Pattison JR. The effect of ischemic preconditioning on repeated sprint cycling performance. Med Sci Sports Exerc. 2015;47(8):1652-8. https://doi.org/10.1249/ mss.0000000000000576

7. Sabino-Carvalho JL, Lopes TR, Obeid-Freitas T, Ferreira TN, Succi JE, Silva AC, et al. Effect of Ischemic Preconditioning on Endurance Performance Does Not Surpass Placebo. Med Sci Sports Exerc. 2017;49(1):124-32. https://doi.org/10.1249/ mss.0000000000001088

8. Slysz JT, Burr JF. Impact of 8 weeks of repeated ischemic preconditioning on running performance. Eur J Appl Physiol. 2019;119(6):1431-7. https://doi.org/10.1007/s00421-019-04133-6

9. Bailey TG, Jones H, Gregson W, Atkinson G, Cable NT, Thijssen DHJ. Effect of ischemic preconditioning on lactate accumulation and running performance. Med Sci Sports Exerc. 2012;44(11):2084-9. https://doi.org/10.1249/ mss.0b013e318262cb17

10. Jean-St-Michel E, Manlhiot C, Li J, Tropak M, Michelsen MM, Schmidt MR, et al. Remote preconditioning improves maximal performance in highly trained athletes. Med Sci Sports Exerc. 2011;43(7):1280-6. https://doi.org/10.1249/ mss.0b013e318206845d

11. Maulik N. Ischemic Preconditioning Mediated Angiogenic Response in the Heart. Antioxidants Redox Signal. 2004;6(2):41321. https://doi.org/10.1089/152308604322899486

12. Bentley DJ, Newell J, Bishop D. Incremental exercise test design and analysis: implications for performance diagnostics in endurance athletes. Sports Med. 2007;37(7):575-86. https://doi. org/10.2165/00007256-200737070-00002
13. Arriel RA, Souza HLR, Silva BVC, Marocolo M. Ischemic preconditioning delays the time of exhaustion in cycling performance during the early but not in the late phase. Motriz: rev educ fis. 2019;25(1):e101821. https://dx.doi.org/10.1590/ s1980-6574201800040050

14. Ferreira TN, Sabino-Carvalho JLC, Lopes TR, Ribeiro IC, Succi JE, Silva AC, et al. Ischemic Preconditioning and Repeated Sprint Swimming: A Placebo and Nocebo Study. Med Sci Sports Exerc. 2016;48(10):1967-75. https://doi.org/10.1249/ mss.0000000000000977

15. Page MJ, McKenzie JE, Bossuyt PM, Boutron I, Hoffmann TC, Mulrow CD, et al. The PRISMA 2020 statement: An updated guideline for reporting systematic reviews. BMJ. 2021;372:n71. http://dx.doi.org/10.1136/bmj.n71

16. Carvalho APV, Silva V GA. Avaliação do risco de viés de ensaios clínicos randomizados pela ferramenta da colaboração Cochrane. Diagn Tratamento [Internet]. 2013;18(1):38-44. Disponível em: http://pesquisa.bvsalud.org/portal/resource/pt/lil-670595\#

17. De Groot PCE, Thijssen DHJ, Sanchez M, Ellenkamp R, Hopman MTE. Ischemic preconditioning improves maximal performance in humans. Eur J Appl Physiol. 2010;108(1):141-6. https://doi. org/10.1007/s00421-009-1195-2

18. Paradis-Deschênes $P$, Joanisse $D R$, Billaut $F$. Ischemic Preconditioning Improves Time Trial Performance at Moderate Altitude. Med Sci Sports Exerc. 2018;50(3):533-41. https://doi. org/10.1249/mss.0000000000001473

19. Kilding AE, Sequeira GM, Wood MR. Effects of ischemic preconditioning on economy, VO2 kinetics and cycling performance in endurance athletes. Eur J Appl Physiol. 2018;118(12):2541-9. http://dx.doi.org/10.1007/s00421-018-3979$\underline{8}$

20. Lehance C, Bury T. Evaluation de la puissance aérobie en laboratoire: Interprétation et implications pratiques pour le médecin du sport. Rev Med Liege [Internet]. 2008;63(7-8):500-3. Disponível em: https://www.rmlg.ulg.ac.be/show.php

21. Burnley M, Jones AM. Oxygen uptake kinetics as a determinant of sports performance. Eur J Sport Sci. 2007;7(2):63-79. https:// doi.org/10.1080/17461390701456148

22. Cruz RSO, Aguiar RA, Turnes T, Pereira KL, Caputo F. Effects of ischemic preconditioning on maximal constant-load cycling performance. J Appl Physiol. 2015;119(9):961-7. https://doi. org/10.1152/japplphysiol.00498.2015 
23. Millet GP, Vleck VE, Bentley DJ. Physiological differences between cycling and running: lessons from triathletes. Sports Med. 2009;39(3):179-206. https://doi.org/10.2165/00007256200939030-00002

24. Bailey TG, Birk GK, Cable NT, Atkinson G, Green DJ, Jones H, et al. Remote ischemic preconditioning prevents reduction in brachial artery flow-mediated dilation after strenuous exercise. Am J Physiol - Hear Circ Physiol. 2012;303(5):H533-8. https://doi. org/10.1152/ajpheart.00272.2012
25. Joyner MJ, Proctor DN. Muscle blood flow during exercise: The limits of reductionism. Med Sci Sports Exerc. 1999;31(7):1036-40. https://doi.org/10.1097/00005768-199907000-00017 